\title{
Crouzon disease
}

INSERM

\section{Source}

INSERM. (1999). Orphanet: an online rare disease and orphan drug data base. Crouzon

disease. ORPHA:207

Crouzon disease is characterized by craniosynostosis and facial hypoplasia. 